\title{
Alternate Mediterranean diet score is positively associated with skeletal muscle mass index in middle-aged adults
}

\author{
Hui-yuan Tian†, Rui Qiu†, Li-peng Jing, Zhan-yong Chen, Geng-dong Chen and Yu-ming Chen* \\ Guangdong Provincial Key Laboratory of Food, Nutrition, and Health, School of Public Health, Sun Yat-sen University, \\ Guangzhou, Guangdong 510080, People's Republic of China
}

(Submitted 12 October 2016 - Final revision received 12 April 2017 - Accepted 18 April 2017- First published online 18 May 2017)

\section{Abstract}

Researches have suggested Mediterranean diet might lower the risk of chronic diseases, but data on skeletal muscle mass (SMM) are limited. This community-based cross-sectional study examined the association between the alternate Mediterranean diet score (aMDS) and SMM in 2230 females and 1059 males aged 40-75 years in Guangzhou, China. General information and habitual dietary information were assessed in face-to-face interviews conducted during 2008-2010 and 3 years later. The aMDS was calculated by summing the dichotomous points for the items of higher intakes of whole grain, vegetables, fruits, legumes, nuts, fish and ratio of MUFA:SFA, lower red meat and moderate ethanol consumption. The SMM of the whole body, limbs, arms and legs were measured using dual-energy X-ray absorptiometry during 2011-2013. After adjusting for potential covariates, higher aMDS was positively associated with skeletal muscle mass index $\left(\mathrm{SMI}, \mathrm{SMM} / \mathrm{height}{ }^{2}, \mathrm{~kg} / \mathrm{m}^{2}\right)$ at all of the studied sites in males (all $P_{\text {trend }}<0.05$ ). The multiple covariate-adjusted SMI means were $2 \cdot 70 \%$ (whole body), $2 \cdot 65 \%$ (limbs), $2.50 \%$ (arms) and $2.70 \%$ (legs) higher in the high ( $v$. low) category aMDS in males (all $P<0.05$ ). In females, the corresponding values were $1.35 \%$ $\left(P_{\text {trend }}=0.03\right), 1.05,0.52$ and $1.20 \%$, $\left(P_{\text {trend }}>0.05\right)$. Age-stratified analyses showed that the favourable associations tended to be more pronounced in the younger subjects aged less than the medians of 59.2 and $62 \cdot 2$ years in females and males $\left(P_{\text {interaction }}>0 \cdot 10\right)$. In conclusion, the aMDS shows protective associations with SMM in Chinese adults, particularly in male and younger subjects.

\section{Key words: Mediterranean diet: Muscle mass: Skeletal muscle mass index: Chinese adults}

Skeletal muscle mass (SMM) increases up to a peak in the fourth decade of life, after which it gradually declines ${ }^{(1)}$. The age-related decrease in SMM is associated with poor health consequences like weakness, frailty ${ }^{(2)}$ and mortality ${ }^{(3)}$, and is an important criterion for sarcopenia, the progressive and generalised loss of SMM and muscle strength or performance ${ }^{(4)}$. The prevalence of sarcopenia has reached an epidemic proportion and costed seriously in terms of both healthcare and human life. In the USA, it is estimated that cost-related health consequences of sarcopenia is over $\$ 18.5$ billion in $2000^{(5)}$. The prevalence of sarcopenia in older community-dwelling Taiwan adults aged 65 years and above is $3.9-7.3 \%$ according to different definitions of sarcopenia ${ }^{(6)}$. Therefore, identification of modifiable risk factors for SMM decline is important to allow more effective prevention of this trend.

The Mediterranean diet (MD) is characterised by high consumption of olive oil, fruit, vegetables, legumes, nuts and cereals, low intakes of SFA and red meat, and moderate intake of $_{\text {alcohol }}^{(7)}$. A large number of studies have showed the MD as a model of healthy eating due to its benefits to the prevention of various chronic diseases, such as $\mathrm{CVD}^{(8)}$, Alzheimer's dementia $^{(9)}$, cognitive decline ${ }^{(10)}$, stroke, depression ${ }^{(11)}$ and cancer $^{(12-15)}$. A few studies have also shown favourable associations between the main components of the MD and the occurrence of sarcopenia, such as moderate consumption of ethanol $^{(16)}$, lower intake of SFA ${ }^{(17)}$ and higher intake of fish oil $^{(18)}$ and fruits and vegetables ${ }^{(19)}$. Previous studies have reported that closer adherence to the MD is associated with lower concentration of inflammation markers ${ }^{(20)}$ and lower oxidative stress $^{(21)}$, from which it might be assumed that there is a positive association between the MD pattern and SMM.

To date, favourable associations between the MD pattern (or score) and with the measures related to SMM were observed in two cross-sectional studies in UK females ${ }^{(22)}$ and in German females (not males) ${ }^{(23)}$, but not in another one in Iranian females ${ }^{(24)}$. One prospective study showed no significant crosssectional nor longitudinal association between the MD score (MDS) and sarcopenia in Hong Kong older adults ${ }^{(25)}$. Among these studies, percent fat-free mass was used in the UK study ${ }^{(22)}$, whereas a ratio of appendicular lean mass:BMI was

Abbreviations: aMDS, alternate Mediterranean diet score; DXA, dual energy X-ray absorptiometry; MD, Mediterranean diet; MDS, MD score; SMI, skeletal muscle mass index; SMM, skeletal muscle mass.

* Corresponding author: Y.-m. Chen, fax +86 20 87330446, email chenyum@mail.sysu.edu.cn

$\dagger$ These authors contributed equally to this work. 
used in the German study ${ }^{(23)}$, and the Iranian study only analysed a posteriori pattern (a MD-like pattern) rather than the MDS. Therefore, the association between MDS and SMM remains speculative. In this cross-sectional study, we examined the hypothesis that higher alternate Mediterranean diet score (aMDS) is associated with greater SMM in a middle-aged and older population.

\section{Methods \\ Study population}

This study was based on cross-sectional data from the Guangzhou Nutrition and Health Study (GNHS), a communitybased prospective cohort study designed to investigate the determinants of major health problems. The cohort study recruited a total of 3169 apparently healthy participants aged 40-75 years old by advertisement and referral between 2008 and 2010 in Guangzhou, China. In the first follow-up survey conducted between 2011 and 2013, 649 participants dropped out and another 879 new participants were recruited in the same way in 2013. Information on habitual diet and covariates were collected in both 2008-2010 and 2011-2013, and SMM was measured by dual energy X-ray absorptiometry (DXA) during the 2011-2013 survey. Details of the study population have been described previously ${ }^{(26)}$. Overall, 110 individuals were excluded due to (I) core data missing (sixty-seven subjects); (II) a history of malignancy (thirty subjects), hepatic cirrhosis (three subjects), and renal insufficiency (six subjects); (III) extreme energy intake ${ }^{(26)}$ (three subjects); and (IV) extreme BMI (one subject). Finally, the study included 3289 participants with at least cross-sectional diet and SMM data collected during 2011-2013. All of the participants provided written informed consent. The study was approved by Sun Yat-Sen University's School of Public Health Ethics Committee and was conducted according to the Declaration of Helsinki.

\section{Data collection}

Information about socio-demographic characteristics (e.g. age, sex, household income, education,), lifestyle (physical activity, smoking and drinking status), use of oral oestrogen and years since menopause (YSM) for females, and personal and family history of chronic diseases and medications was collected by means of face-to-face interviews using a structured questionnaire. Physical activity was specified as activities of daily life, work time and leisure time (excluding sleeping and sitting) and was calculated as the metabolic equivalent $(\mathrm{h} / \mathrm{d})$ as described previously ${ }^{(27)}$. Height and weight were measured with the participant in a standing position and wearing light clothes and no shoes.

\section{Assessment of dietary intake}

Participants were asked about their food intake at five possible frequencies (never, per year, per month, per week and per day) over the past year using a seventy-nine-item quantitative FFQ. The Chinese Food Composition Table, 2004 was used to calculate the average daily intake of nutrients and total energy ${ }^{(28)}$.
The interview methods have been described in detail elsewhere $^{(26)}$. For the 2520 subjects, who provided dietary data at both baseline and the first follow-up, we calculated the average intake, which is commonly used to assess long-term dietary habits and reduce within-subject variation as did in previous studies ${ }^{(29,30)}$; for the 879 new participants, only one set of dietary data was available and used in further analyses.

\section{Calculation of alternate Mediterranean diet score}

The traditional MDS was developed by Trichopoulou et al. ${ }^{(31)}$ to indicate the adherence to traditional MD in a Greek population, in which eight items were included and classified adherence by assigning 0 to 5 points for each food component: high MUFA:SFA ratio; moderate consumption of ethanol; high consumption of cereals, fruits, vegetables, legumes; low consumption of milk and dairy products, meat and meat products. Fung et al.'s aMDS ${ }^{(32)}$ included vegetables, fruits, nuts, whole grains, legumes, fish, ratio of MUFA:SFA, red and processed meats and alcohol. The aMDS is calculated by summing the dichotomous points ( 0 or 1 ) for the items of higher intakes of whole grain, vegetables (excluding potatoes), fruits, legumes, nuts, fish and ratio of MUFA:SFA, lower red meat according to their sex-specific group medians, and moderate ethanol consumption $(5-15 \mathrm{~g} / \mathrm{d} \text { in females and } 15-25 \mathrm{~g} / \mathrm{d} \text { in males) })^{(32)}$ (online Supplementary Table S1). The possible score range for aMDS was 0 to 9, with a higher score representing closer adherence to the $\mathrm{MD}^{(32)}$. As previous studies suggested that SMM might be associated with fish oil ${ }^{(33)}$ and nut ${ }^{(34)}$, we finally chose to use Fung et al.'s aMDS to evaluate the diet quality in our study.

\section{Muscle mass assessment}

During the 2011-2013 survey, the SMM (kg) of the whole body and the arms and legs was measured by DXA (Discovery W; Hologic Inc.) using a default model and analysed with Hologic Discovery software version 3.2. The DXA scanner was calibrated daily. The in vivo coefficients of variation of the duplicated measurements in thirty participants after repositioning were $6 \cdot 1 \%$ for the whole body, $3 \cdot 1 \%$ for the limbs, $2 \cdot 2 \%$ for the arms and $4.0 \%$ for the legs. The skeletal muscle mass index (SMI) was defined as SMM $/$ height $^{2}$ (in $\left.\mathrm{kg} / \mathrm{m}^{2}\right)^{(4)}$, and SMI were calculated for the whole body, four limbs, arms and legs, respectively.

\section{Statistical analysis}

Analyses were performed using SPSS (version 17.0; SPSS Inc.), and two-sided $P$ values below 0.05 were considered significant. The participants were divided into three categories (low (0-3), middle $(4,5)$ and high $(6-8)$ ) according to the aMDS. The common characteristics of the participants were presented as frequencies and percentages for categorical variables, and mean values and standard deviations for continuous variables by sex. The $t$ test, $\chi^{2}$ test and Wilcoxon rank sum test were used to compare the differences between females and males for the continuous, categorical and ordinal variables, respectively. 
Multivariate ANCOVA were used to compare the covariateadjusted mean SMI between the three aMDS categories in females and males separately. Model 1 adjusted for age and physical activity. Model 2 adjusted for age, physical activity, smoking status, passive smoking status, dairy foods, daily energy intake and dietary protein intake in males, and further adjusted for YSM and oral oestrogen use in females based on theoretical relevance and significance in univariate analysis $(P<0 \cdot 2)$. Furthermore, we carried out age stratified analyses according to the group medians of age in males ( 62.2 years) and females (59.2 years) using model 2. Bonferroni tests were used for multiple comparisons between the aMDS categories. Our study could achieve a statistical power of $86 \%$ to detect a non-zero contrast of the means of the total body SMI based on the our data in total subjects using PASS $2011^{(35)}$.

In sensitive analyses, regression analysis was used to test whether there was a significant linear association when the aMDS was considered as a continuous variable. Moreover, we compared the SMI by dichotomous categories of each component in model 2 to assess which components of the aMDS were responsible for the associations of aMDS. We also performed discriminant classification analysis using Fisher's linear discriminant function and the calculation of Wilks' $\lambda$ to explore the discriminating ability of the individual components of the aMDS. To explore whether the association between aMDS and SMI was modified by age, sex, physical activity or dietary intake of energy and protein, the potential interactions were assessed in model 2 .

\section{Results}

The characteristics of the study population are shown in Table 1. Analyses included 2225 females and 1064 males. Sex, BMI, energy intake, smoking status, physical activity and food intakes were significantly different across the three aMDS categories.

Comparisons of the covariate-adjusted mean values with their standard errors of the SMI by aMDS categories are presented in Table 2. Generally, there is a favourable association between aMDS and SMI. However, the favourable associations tended to be more pronounced in males (all $P_{\text {trend }}<0.05$ ) than in females at all measured sites in both models 1 and 2. In males, graded and positive associations were found between the aMDS and SMI at all measured sites (all $P_{\text {trend }}<0 \cdot 05$ ). After adjusting for multiple covariates in model 2 , the favourable associations tended to be slightly strengthened (all $P_{\text {trend }}$ 0.002-0.013).

Table 1. Characteristics of the participants by categories of the alternate Mediterranean diet score (aMDS) (Mean values and standard deviations; numbers and percentages)

\begin{tabular}{|c|c|c|c|c|c|c|c|}
\hline \multirow[b]{2}{*}{ aMDS } & \multicolumn{2}{|c|}{ Low (0-3) } & \multicolumn{2}{|c|}{ Middle $(4,5)$} & \multicolumn{2}{|c|}{ High (6-8) } & \multirow[b]{2}{*}{$P$} \\
\hline & Mean & SD & Mean & SD & Mean & SD & \\
\hline$n$ & \multicolumn{2}{|c|}{1283} & \multicolumn{2}{|c|}{1377} & \multicolumn{2}{|c|}{629} & \\
\hline Female & & & & & & & 0.783 \\
\hline$n$ & \multicolumn{2}{|c|}{857} & \multicolumn{2}{|c|}{942} & \multicolumn{2}{|c|}{431} & \\
\hline$\%$ & \multicolumn{2}{|c|}{67.1} & \multicolumn{2}{|c|}{$68 \cdot 1$} & \multicolumn{2}{|c|}{68.5} & \\
\hline Age (years) ${ }^{\star}$ & 60.49 & 6.07 & $60 \cdot 66$ & 5.86 & 60.92 & 5.94 & 0.335 \\
\hline Body fat percentage & 32.40 & 6.65 & 32.40 & $6 \cdot 64$ & $32 \cdot 31$ & 6.51 & 0.955 \\
\hline Energy $(\mathrm{kJ} / \mathrm{d})$ & 6770 & 1678 & 6707 & 1623 & 6736 & 1707 & 0.650 \\
\hline Energy (kcal/d) & 1618 & 401 & 1603 & 388 & 1610 & 408 & 0.650 \\
\hline Smokingt & & & & & & & 0.001 \\
\hline$n$ & \multicolumn{2}{|c|}{183} & \multicolumn{2}{|c|}{155} & \multicolumn{2}{|c|}{54} & \\
\hline$\%$ & \multicolumn{2}{|c|}{$14 \cdot 3$} & \multicolumn{2}{|c|}{$11 \cdot 2$} & \multicolumn{2}{|c|}{$8 \cdot 6$} & \\
\hline Passive smoking $\ddagger$ & & & & & & & 0.001 \\
\hline$n$ & \multicolumn{2}{|c|}{336} & \multicolumn{2}{|c|}{308} & \multicolumn{2}{|c|}{121} & \\
\hline$\%$ & \multicolumn{2}{|c|}{$26 \cdot 3$} & \multicolumn{2}{|c|}{$22 \cdot 3$} & \multicolumn{2}{|c|}{$19 \cdot 2$} & \\
\hline Years since menopause & $9 \cdot 77$ & $6 \cdot 13$ & 9.47 & 5.81 & 9.49 & 6.02 & 0.523 \\
\hline Oestrogen use & & & & & & & 0.210 \\
\hline$n$ & \multicolumn{2}{|c|}{40} & \multicolumn{2}{|c|}{59} & \multicolumn{2}{|c|}{29} & \\
\hline$\%$ & & & & & & & \\
\hline Physical activity (MET h/d)§ & $16 \cdot 44$ & 6.74 & 16.99 & 6.86 & 17.58 & 7.04 & 0.002 \\
\hline Protein intake $(\mathrm{g} / \mathrm{d})$ & 67.40 & $10 \cdot 14$ & $70 \cdot 16$ & $10 \cdot 33$ & $72 \cdot 00$ & 9.80 & $<0.001$ \\
\hline Components of aMDS (serving/d & & & & & & & \\
\hline Whole grain (serving/d) & 0.22 & 0.33 & 0.32 & 0.31 & 0.40 & 0.24 & $<0.001$ \\
\hline Vegetables (except potatoes) & 2.85 & 1.00 & 3.56 & 1.21 & 4.24 & 1.21 & $<0.001$ \\
\hline Fruits (included juices) & $1 \cdot 18$ & 0.84 & 1.60 & 0.88 & 1.98 & 0.84 & $<0.001$ \\
\hline Legumes & 0.26 & 0.40 & 0.39 & 0.29 & 0.48 & 0.30 & $<0.001$ \\
\hline Nuts & 0.07 & 0.09 & $0 \cdot 11$ & 0.11 & 0.14 & 0.11 & $<0.001$ \\
\hline Fish & 0.75 & 0.50 & 1.04 & 0.83 & 1.25 & 0.67 & $<0.001$ \\
\hline Red meat & 1.73 & 0.74 & 1.39 & 0.64 & $1 \cdot 16$ & 0.67 & $<0.001$ \\
\hline Ratio of MUFA:SFA & 1.40 & 0.14 & 1.44 & $0 \cdot 14$ & 1.47 & 0.13 & $<0.001$ \\
\hline Ethanol ( $\mathrm{g} / \mathrm{month})$ & 0.65 & 4.86 & 0.76 & $5 \cdot 34$ & 0.37 & $2 \cdot 24$ & 0.234 \\
\hline
\end{tabular}

MET, metabolic equivalent.

* Calculated by the time of dual-energy X-ray absorptiometry examination.

$\dagger$ Smoking was defined if they had smoked at least five packages of cigarettes during the last year.

‡ Passive smoking was defined if they had been exposed to others' tobacco smoke for at least 5 min or one cigarette per day for the past year.

$\S$ Physical activities (MET h/d) included daily activities in occupation, leisure-time and household-chores apart from the lying and sitting. 
Table 2. Comparisons of covariate-adjusted skeletal muscle mass index (SMI) by alternate Mediterranean diet score (aMDS) categories in males and females (Mean values with their standard errors)

\begin{tabular}{|c|c|c|c|c|c|c|c|c|c|}
\hline & \multicolumn{6}{|c|}{ Categories of aMDS } & \multirow[b]{3}{*}{$\%$ difference (high $v$. low) $\dagger$} & \multirow{2}{*}{\multicolumn{2}{|c|}{ ANCOVA }} \\
\hline & \multicolumn{2}{|c|}{ Low (0-3) } & \multicolumn{2}{|c|}{ Middle $(4,5)$} & \multicolumn{2}{|c|}{ High (6-8) } & & & \\
\hline & Mean & SEM & Mean & SEM & Mean & SEM & & $P_{\text {difference }}$ & $P_{\text {trend }} \neq$ \\
\hline \multicolumn{10}{|l|}{ Males } \\
\hline$n$ & \multicolumn{2}{|c|}{427} & \multicolumn{2}{|c|}{437} & \multicolumn{2}{|c|}{200} & & & \\
\hline \multicolumn{10}{|c|}{ Model 1, SMI $\left(\mathrm{kg} / \mathrm{m}^{2}\right) \S$} \\
\hline Total body & 16.52 & 0.08 & $16 \cdot 77$ & 0.08 & 16.91 & $0.11^{*}$ & 2.41 & 0.006 & 0.004 \\
\hline Limbs & 7.37 & 0.04 & 7.49 & 0.04 & 7.54 & $0.05^{\star}$ & $2 \cdot 27$ & 0.018 & 0.011 \\
\hline Arms & 1.88 & 0.01 & 1.92 & $0.01^{*}$ & 1.92 & 0.02 & $2 \cdot 18$ & 0.006 & 0.025 \\
\hline Legs & 5.49 & 0.03 & 5.56 & 0.03 & 5.62 & $0.04^{*}$ & 2.29 & 0.033 & 0.013 \\
\hline \multicolumn{10}{|c|}{ Model 2, SMI $\left(\mathrm{kg} / \mathrm{m}^{2}\right) \|$} \\
\hline Total body & 16.49 & 0.08 & $16 \cdot 78$ & $0.08^{*}$ & 16.94 & $0 \cdot 11^{\star *}$ & $2 \cdot 70$ & 0.003 & 0.002 \\
\hline Limbs & 7.36 & 0.04 & 7.49 & $0.04^{\star}$ & 7.56 & $0.06^{*}$ & 2.65 & 0.007 & 0.004 \\
\hline Arms & 1.88 & 0.01 & 1.92 & $0.01^{* *}$ & 1.92 & $0.02^{*}$ & 2.50 & 0.003 & 0.013 \\
\hline Legs & 5.49 & 0.03 & 5.57 & 0.03 & 5.63 & $0.04^{*}$ & $2 \cdot 70$ & 0.013 & 0.005 \\
\hline \multicolumn{10}{|l|}{ Females } \\
\hline & \multicolumn{2}{|c|}{856} & \multicolumn{2}{|c|}{940} & \multicolumn{2}{|c|}{429} & & & \\
\hline \multicolumn{10}{|c|}{ Model 1, SMI $\left(\mathrm{kg} / \mathrm{m}^{2}\right) \S$} \\
\hline Total body & 14.03 & 0.05 & 14.05 & 0.05 & $14 \cdot 17$ & 0.07 & 0.96 & 0.258 & 0.119 \\
\hline Limbs & 5.95 & 0.02 & 5.96 & 0.02 & 5.99 & 0.03 & 0.71 & 0.603 & 0.317 \\
\hline Arms & 1.36 & 0.01 & 1.36 & 0.01 & 1.36 & 0.01 & 0.15 & 0.960 & 0.875 \\
\hline Legs & 4.59 & 0.02 & 4.60 & 0.02 & 4.63 & 0.03 & 0.87 & 0.475 & 0.228 \\
\hline \multicolumn{10}{|c|}{ Model 2, SMI $\left(\mathrm{kg} / \mathrm{m}^{2}\right) \|$} \\
\hline Total body & 14.00 & 0.05 & 14.04 & 0.05 & $14 \cdot 19$ & 0.07 & 1.35 & 0.088 & 0.030 \\
\hline Limbs & 5.93 & 0.02 & 5.95 & 0.02 & 5.99 & 0.03 & 1.05 & 0.332 & 0.138 \\
\hline Arms & 1.35 & 0.01 & 1.36 & 0.01 & 1.36 & 0.01 & 0.52 & 0.743 & 0.523 \\
\hline Legs & 4.58 & 0.02 & 4.60 & 0.02 & 4.63 & 0.03 & 1.20 & 0.255 & 0.098 \\
\hline
\end{tabular}

The SMI means were $2.70 \%$ (whole body), $2.65 \%$ (limbs), $2.50 \%$ (arms) and $2.70 \%$ (legs) higher in the high ( $v$. low) aMDS category (all $P<0 \cdot 05$ ), respectively. In females, the corresponding values were $1.35 \%$ (whole body), $1.05 \%$ (limbs), $0.52 \%$ (arms) and $1.20 \%$ (legs), but not statistically significant except the whole body $\left(P_{\text {trend }}=0.03\right)$. Age-stratified results showed that the favourable associations tended to be more substantial in younger participants than in the older subjects in both males and females,. However, no statistically significant interaction was observed in males $(P: 0 \cdot 516-0.821)$ and in females (P: 0.109-0.706) (Table 3).

Table 4 showed the associations between food components of the aMDS and SMI in male and female participants (model 2). In males, food components with significant associations included vegetables (all sites, $P_{\text {trend }}$ 0.003-0.030), fruits (all sites, $P_{\text {trend }}: 0 \cdot 014-0.022$ ), red and processed meats (all sites, $P_{\text {trend }}: 0 \cdot 001-0 \cdot 011$ ), fish (total body, $P_{\text {trend }}: 0 \cdot 011$ ) and ratio of MUFA:SFA (arms, $P_{\text {trend: }}$ 0.017). In female, it is vegetables and fruits (all sites except arms, $P_{\text {trend }}$ : <0.001-0.005). In consistent with the results in Table 4 , discriminant classification analysis showed that the components with significant discriminating ability were mainly those of vegetable, fruit and ratio of MUFA: SFA (online Supplementary Table S2).

Multiple regression analyses showed significant linear relationships between the aMDS (as a continuous variable) and
SMI at all studied sites in males $(P$ : $<0.001-0.036)$, and at all studied sites (except for the arms) in females $(P:<0 \cdot 001-0 \cdot 302)$ (online Supplementary Table S3). We examined whether the association between aMDS and SMI was modified by smoking, passive smoking, physical activity, dietary intake of total energy and protein. No significant interactions were observed

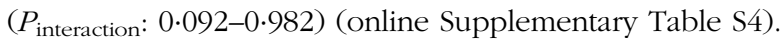

\section{Discussion}

In this cross-sectional study of a middle-aged and elderly Chinese population, a favourable association was found between aMDS and SMI after adjusting for potential confounding variables. This association tended to be more pronounced in males ( $v$. females) and in younger ( $v$. older) subjects with ages less than the median age of $59 \cdot 2$ and $62 \cdot 2$ years in females and males.

Previous studies have suggested favourable associations between some components of the MD diet (e.g. moderate consumption of alcohol ${ }^{(16)}$, lower intake of SFA ${ }^{(17)}$ and higher intake of fish oil ${ }^{(18)}$ and fruits and vegetables $\left.{ }^{(19)}\right)$ and the risk of sarcopenia. Analysing dietary data as a whole rather than single nutrient or single food has been viewed as an important method to nutritional epidemiologists. As foods were eaten together, dietary pattern analysis or quality scores could be easier for 
Table 3. Age and sex stratified comparisons of covariate-adjusted skeletal muscle mass index (SMI) by categories of alternate Mediterranean diet score $($ aMDS)*

(Mean values with their standard errors)

\begin{tabular}{|c|c|c|c|c|c|c|c|c|c|c|}
\hline & \multicolumn{6}{|c|}{ Categories of aMDS } & \multirow[b]{3}{*}{ \% difference (C3 v. C1)† } & \multirow{2}{*}{\multicolumn{2}{|c|}{ ANCOVA }} & \multirow[b]{3}{*}{$P_{\text {interaction }}$} \\
\hline & \multicolumn{2}{|c|}{ Low (0-3) } & \multicolumn{2}{|c|}{ Middle $(4,5)$} & \multicolumn{2}{|c|}{ High (6-8) } & & & & \\
\hline & Mean & SEM & Mean & SEM & Mean & SEM & & $P_{\text {difference }}$ & $P_{\text {trend }} \ddagger$ & \\
\hline \multicolumn{11}{|l|}{ Males } \\
\hline \multicolumn{11}{|l|}{$<62.2$ years } \\
\hline$n$ & \multicolumn{2}{|c|}{231} & \multicolumn{2}{|c|}{204} & \multicolumn{2}{|c|}{99} & & & & \\
\hline Total-SMI & $16 \cdot 65$ & 0.11 & $16 \cdot 86$ & 0.11 & $17 \cdot 07$ & 0.17 & $2 \cdot 51$ & 0.107 & 0.040 & 0.760 \\
\hline Appendicular SMI & $7 \cdot 44$ & 0.05 & 7.55 & 0.06 & $7 \cdot 68$ & 0.08 & $3 \cdot 10$ & 0.063 & 0.021 & 0.588 \\
\hline Arms SMI & 1.90 & 0.01 & 1.95 & 0.02 & 1.96 & 0.02 & 2.89 & 0.037 & 0.043 & 0.821 \\
\hline Legs SMI & 5.54 & 0.04 & $5 \cdot 60$ & 0.04 & $5 \cdot 72$ & 0.06 & $3 \cdot 18$ & 0.077 & 0.024 & 0.516 \\
\hline \multicolumn{11}{|l|}{$\geq 62.2$ years } \\
\hline$n$ & \multicolumn{2}{|c|}{196} & \multicolumn{2}{|c|}{233} & \multicolumn{2}{|c|}{101} & & & & \\
\hline Total-SMI & $16 \cdot 35$ & 0.12 & $16 \cdot 68$ & $0 \cdot 10$ & $16 \cdot 80$ & 0.16 & 2.78 & 0.036 & 0.023 & \\
\hline Appendicular SMI & $7 \cdot 28$ & 0.06 & $7 \cdot 42$ & 0.05 & $7 \cdot 44$ & 0.08 & $2 \cdot 14$ & 0.115 & 0.097 & \\
\hline Arms SMI & 1.86 & 0.02 & 1.90 & 0.01 & 1.89 & 0.02 & 1.89 & 0.140 & 0.196 & \\
\hline Legs SMI & 5.43 & 0.04 & $5 \cdot 53$ & 0.04 & $5 \cdot 55$ & 0.06 & $2 \cdot 23$ & 0.135 & 0.092 & \\
\hline \multicolumn{11}{|l|}{ Females } \\
\hline \multicolumn{11}{|l|}{$<62.2$ years } \\
\hline$n$ & \multicolumn{2}{|c|}{410} & \multicolumn{2}{|c|}{493} & \multicolumn{2}{|c|}{215} & & & & \\
\hline Total-SMI & 13.94 & 0.07 & 13.97 & 0.07 & $14 \cdot 19$ & 0.10 & 1.80 & 0.113 & 0.045 & 0.706 \\
\hline Appendicular SMI & 5.89 & 0.04 & $5 \cdot 92$ & 0.03 & 6.03 & 0.05 & $2 \cdot 26$ & 0.076 & 0.027 & 0.175 \\
\hline Arms SMI & 1.34 & 0.01 & 1.35 & 0.01 & $1 \cdot 36$ & 0.01 & 1.42 & 0.376 & 0.198 & 0.611 \\
\hline Legs SMI & 4.56 & 0.03 & 4.57 & 0.03 & 4.67 & 0.04 & 2.50 & 0.045 & 0.018 & 0.109 \\
\hline \multicolumn{11}{|l|}{$\geq 62.2$ years } \\
\hline$n$ & \multicolumn{2}{|c|}{446} & \multicolumn{2}{|c|}{447} & \multicolumn{2}{|c|}{214} & & & & \\
\hline Total-SMI & 14.06 & 0.07 & $14 \cdot 12$ & 0.07 & $14 \cdot 20$ & $0 \cdot 10$ & 0.99 & 0.510 & 0.256 & \\
\hline Appendicular SMI & 5.96 & 0.03 & 6.00 & 0.03 & 5.96 & 0.05 & 0.03 & 0.682 & 0.975 & \\
\hline Arms SMI & 1.37 & 0.01 & 1.37 & 0.01 & $1 \cdot 36$ & 0.01 & 0.02 & 0.944 & 0.841 & \\
\hline Legs SMI & 4.60 & 0.03 & 4.63 & 0.03 & 4.60 & 0.04 & 0.02 & 0.603 & 0.981 & \\
\hline
\end{tabular}

* All analyses were controlled for age, physical activity, smoking status, passive smoking status, daily energy intake and dietary protein intake, and years since menopause and oral oestrogen (in females only) based on theoretical relevance and significance in univariate analysis $(P<0.2)$.

$\dagger$ Percentage difference $=($ high - low $) /$ low $\times 100 \%$.

$\ddagger P$ value for linear trend.

making recommendations and to avoid the complex interactions among different kinds of food. Furthermore, eating patterns seem more efficient in the association analyses between dietary factors and nutrition-related diseases ${ }^{(36)}$. To date, however, few studies have examined the association between the MDS and muscle mass or sarcopenia risk.

One cross-sectional study reported significant positive associations between the MDS and fat-free mass\% in 2570 healthy females aged 18-79 years from the Twins UK study ${ }^{(22)}$. The Berlin Aging Study II, a cross-sectional study including 1509 participants (51\% females, 68.2 (SD 3.7) years), showed that greater adherence to the MD was associated with a higher ratio of appendicular lean mass:BMI in females $(P=0.004)$, but not in males ${ }^{(23)}$. Another cross-sectional study showed greater MD-like pattern score was associated with a lower presence of sarcopenia (tertile $3 v$. tertile $1: \mathrm{OR}=0.42 ; 95 \%$ CI $0 \cdot 18,0 \cdot 97$ ) in 300 elderly Iranian males and females ${ }^{(24)}$. However, null associations of the MDS were found with the odds or the 4-year incidence of sarcopenia (low SMI, low grip strength and low gait speed) were not found in either the cross-sectional analysis $(P>0.05)$ or the longitudinal analysis $(P>0.05)$ in a sample of Hong Kong Chinese males ( $n$ 1979) and females ( $n$ 1978) aged 65 years or older, possibly because of the limited number of sarcopenic subjects ( $n$ 290) at baseline and follow-up
(264 new cases) ${ }^{(25)}$. In this community-based study, a favourable association between aMDS and SMI was found in a middleaged and elderly Chinese population, particular in males and younger subjects (age $<$ median). These findings and some previous results highlight the importance and practical value of the MD pattern (or higher MDS) in the maintenance of muscle mass.

Higher aMDS may prevent muscle mass reduction through several mechanisms. First, better adherence to the MD pattern is inversely associated with some pro-inflammatory cytokines, such as C-reactive protein and IL-6. Chronic systemic inflammation was suggested to be one of the key contributors of SMM declination $^{(37)}$. Besides, people with higher aMDS have high intake of fruit and vegetables, which are rich in antioxidants, as oxidative stress is the major mechanism of sarcopenia implicated in aging adults. Sensitivity analysis of aMDS components in our study also showed significant favourable associations between intakes of fruit and vegetables, fruits and SMI, suggesting fruit and vegetables as important explanatory variables.

This study found a more pronounced relationship between aMDS and SMI in males than in females. Similar trends were also found when we consider the aMDS as a continuous variable. Favourable associations between the intake of fruit and vegetables and sarcopenia (defined as the lowest quintile of lean mass) in older adults were similarly more prominent in 
Table 4. Comparisons of covariate-adjusted skeletal muscle mass index (SMI) by groups of food components of the alternate Mediterranean diet score (aMDS) in males and females*

(Mean values with their standard errors)

\begin{tabular}{|c|c|c|c|c|c|c|c|c|c|c|c|c|c|c|c|c|}
\hline & \multicolumn{8}{|c|}{ Males SMI $\left(\mathrm{kg} / \mathrm{m}^{2}\right)$} & \multicolumn{8}{|c|}{ Females SMI $\left(\mathrm{kg} / \mathrm{m}^{2}\right)$} \\
\hline & \multicolumn{2}{|c|}{ Total body } & \multicolumn{2}{|c|}{ Limbs } & \multicolumn{2}{|c|}{ Arms } & \multicolumn{2}{|c|}{ Legs } & \multicolumn{2}{|c|}{ Total body } & \multicolumn{2}{|c|}{ Limbs } & \multicolumn{2}{|c|}{ Arms } & \multicolumn{2}{|c|}{ Legs } \\
\hline & Mean & SEM & Mean & SEM & Mean & SEM & Mean & SEM & Mean & SEM & Mean & SEM & Mean & SEM & Mean & SEM \\
\hline \multicolumn{17}{|l|}{ Vegetables } \\
\hline$\leq$ Median & 16.54 & 0.07 & 7.39 & 0.03 & 1.89 & 0.01 & $5 \cdot 51$ & 0.03 & 13.96 & 0.04 & 5.92 & 0.02 & 1.35 & 0.01 & 4.57 & 0.02 \\
\hline$>$ Median & $16 \cdot 82$ & 0.07 & 7.50 & 0.03 & 1.92 & 0.01 & 5.58 & 0.03 & $14 \cdot 16$ & 0.04 & 6.00 & 0.02 & 1.36 & 0.01 & 4.63 & 0.02 \\
\hline$P_{\text {difference }}$ & \multicolumn{2}{|c|}{0.004} & \multicolumn{2}{|c|}{0.031} & \multicolumn{2}{|c|}{0.014} & \multicolumn{2}{|c|}{0.056} & \multicolumn{2}{|c|}{0.002} & \multicolumn{2}{|c|}{0.011} & \multicolumn{2}{|c|}{0.133} & \multicolumn{2}{|c|}{0.007} \\
\hline$P_{\text {trend }}$ & 0.0 & & & & & & 0.0 & & $<0$. & & 0.0 & & 0.0 & & 0.0 & \\
\hline Legumes & & & & & & & & & & & & & & & & \\
\hline$\leq$ Median & $16 \cdot 64$ & 0.07 & 7.44 & 0.03 & 1.89 & 0.01 & 5.54 & 0.03 & 14.00 & 0.04 & 5.94 & 0.02 & 1.36 & 0.01 & 4.58 & 0.02 \\
\hline$>$ Median & 16.73 & 0.07 & 7.45 & 0.03 & 1.91 & 0.01 & 5.54 & 0.03 & $14 \cdot 12$ & 0.04 & 5.98 & 0.02 & 1.36 & 0.01 & 4.62 & 0.02 \\
\hline$P_{\text {difference }}$ & & & & & & & 0.9 & & & & 0.2 & & 0.6 & & 0.1 & \\
\hline$P_{\text {trend }}$ & 0.2 & & & & & & 0.8 & & & & 0.7 & & 0.6 & & 0.6 & \\
\hline Fruits & & & & & & & & & & & & & & & & \\
\hline$\leq$ Median & 16.59 & 0.07 & 7.39 & 0.33 & 1.89 & 0.01 & $5 \cdot 50$ & 0.03 & 13.96 & 0.04 & 5.91 & 0.02 & 1.35 & 0.01 & 4.56 & 0.02 \\
\hline$>$ Median & $16 \cdot 78$ & 0.07 & 7.50 & 0.33 & 1.91 & 0.01 & $5 \cdot 58$ & 0.03 & $14 \cdot 16$ & 0.04 & $6 \cdot 00$ & 0.02 & 1.36 & 0.01 & 4.64 & 0.02 \\
\hline$P_{\text {difference }}$ & & & & & & & 0.0 & & & & 0.0 & & 0.0 & & 0.0 & \\
\hline$P_{\text {trend }}$ & $0 . c$ & & & & & & 0.0 & & & & 0.0 & & 0.1 & & 0.0 & \\
\hline Nuts & & & & & & & & & & & & & & & & \\
\hline$\leq$ Median & 16.65 & 0.07 & 7.42 & 0.03 & 1.91 & 0.01 & $5 \cdot 52$ & 0.03 & 14.09 & 0.04 & 5.96 & 0.02 & 1.36 & 0.01 & 4.60 & 0.17 \\
\hline$>$ Median & $16 \cdot 72$ & 0.07 & 7.46 & 0.03 & 1.90 & 0.01 & $5 \cdot 56$ & 0.03 & 14.03 & 0.04 & 5.95 & 0.02 & 1.35 & 0.01 & 4.60 & 0.17 \\
\hline$P_{\text {difference }}$ & 0.4 & & & & & & 0.2 & & & & 0.6 & & 0.3 & & $0 . \varepsilon$ & \\
\hline$P_{\text {trend }}$ & 0.2 & & & & & & 0.1 & & & & 0.5 & & 0.3 & & 0.6 & \\
\hline Whole grains & & & & & & & & & & & & & & & & \\
\hline$\leq$ Median & $16 \cdot 73$ & 0.07 & 7.45 & 0.03 & 1.91 & 0.01 & $5 \cdot 54$ & 0.03 & 14.05 & 0.04 & 5.94 & 0.02 & 1.36 & 0.01 & 4.58 & 0.02 \\
\hline$>$ Median & $16 \cdot 66$ & 0.07 & 7.45 & 0.03 & 1.90 & 0.01 & 5.55 & 0.03 & 14.07 & 0.04 & 5.97 & 0.02 & 1.36 & 0.01 & 4.62 & 0.02 \\
\hline$P_{\text {difference }}$ & 0.2 & & & & & & 0.8 & & & & 0.3 & & 0.7 & & 0.1 & \\
\hline$P_{\text {trend }}$ & 0.6 & & & & & & 0.6 & & & & 0.0 & & 0.3 & & 0.0 & \\
\hline Red and proc & essed $m$ & & & & & & & & & & & & & & & \\
\hline$\leq$ Median & 16.86 & 0.07 & 7.54 & 0.03 & 1.92 & 0.01 & $5 \cdot 61$ & 0.03 & $14 \cdot 11$ & 0.04 & 5.98 & 0.02 & 1.37 & 0.01 & 4.62 & 0.02 \\
\hline$>$ Median & $16 \cdot 50$ & 0.07 & 7.35 & 0.03 & 1.88 & 0.01 & 5.47 & 0.03 & 14.02 & 0.04 & 5.93 & 0.02 & 1.35 & 0.01 & 4.58 & 0.02 \\
\hline$P_{\text {difference }}$ & $<0$. & & $<0$ & & & & $<0.0$ & & & & 0.1 & & 0.0 & & 0.2 & \\
\hline$P_{\text {trend }}$ & & & & & & & 0.0 & & & & 0.0 & & 0.0 & & $0 . c$ & \\
\hline Fish & & & & & & & & & & & & & & & & \\
\hline$\leq$ Median & 16.59 & 0.07 & 7.42 & 0.04 & 1.89 & 0.01 & $5 \cdot 53$ & 0.03 & 14.04 & 0.04 & 5.96 & 0.02 & 1.36 & 0.01 & 4.60 & 0.02 \\
\hline$>$ Median & $16 \cdot 78$ & 0.07 & 7.67 & 0.04 & 1.91 & 0.01 & 5.56 & 0.03 & 14.08 & 0.04 & 5.96 & 0.02 & 1.36 & 0.01 & 4.60 & 0.02 \\
\hline$P_{\text {difference }}$ & $0 \cdot C$ & & & & & & 0.4 & & & & 0.9 & & 0.9 & & 0.8 & \\
\hline$P_{\text {trend }}$ & 0.6 & & & & & & 0.1 & & & & 0.2 & & 0.1 & & 0.2 & \\
\hline Ratio of MUF & A:SFA & & & & & & & & & & & & & & & \\
\hline$\leq$ Median & $16 \cdot 61$ & 0.07 & 7.44 & 0.03 & 1.78 & 0.01 & 5.55 & 0.03 & $14 \cdot 11$ & 0.04 & 5.98 & 0.02 & 1.37 & 0.01 & 4.62 & 0.02 \\
\hline$>$ Median & $16 \cdot 76$ & 0.07 & 7.45 & 0.03 & 1.92 & 0.01 & 5.54 & 0.03 & 14.02 & 0.04 & 5.93 & 0.02 & 1.35 & 0.01 & 4.58 & 0.02 \\
\hline$P_{\text {difference }}$ & 0 & & & & & & 0.7 & & & & 0.1 & & 0.0 & & 0.2 & \\
\hline$P_{\text {trend }}$ & $0 . c$ & & & & & & 0.6 & & & & 0.6 & & 0.1 & & 0.3 & \\
\hline
\end{tabular}

* All analyses were controlled for age, physical activity, smoking status, passive smoking status, daily energy intake and dietary protein intake, and years since menopause and oral oestrogen (in females only).

males than in females in the fourth Korea National Health and Nutrition Examination ${ }^{(19)}$. Previous studies have also reported that chronic periods of negative energy balance tend to have less of an effect on growth and loss of body tissue in females than in males ${ }^{(38)}$. Other studies ${ }^{(39-41)}$ have shown much higher rates of reduction in appendicular lean mass in males than in females. Therefore, males appear to be more sensitive than females in terms of muscle mass. The difference in sex hormones may also play a role, as oestrogen could contribute to the antioxidant defense ability in female muscles ${ }^{(42)}$. In the age-stratified analysis, we found that the favourable associations were more pronounced in the younger group than in the older one. The cumulative effect of a long-term adherence to the healthy dietary pattern seemed to be attenuated at the elderly stage. Our findings suggested that the middle aged (but not elderly) period might be the 'window period' for the intervention of dietary pattern on body SMM. Further longitudinal studies are needed to clarify this issue.

This study had several strengths. First, it examined the association between aMDS and SMM in a large communitybased population, adding to the limited evidence. Second, average food intake data were used for analyses, which should provide a better estimation of habitual intake before muscle mass was measured in the majority of participants. Third, SMM was measured by DXA rather than bioelectric impedance. Previous studies have shown the excellent validity of DXA compared with the golden methods of MRI and computed tomography (CT) $\left(R^{2} \text { 0.96-0.98 }\right)^{(43,44)}$. Lastly, SMI was assessed at multiple sites. Total body SMI provide us an overall evaluation of participants' SMM, but when it 
comes to sarcopenia, we always focus on appendicular muscle mass rather than trunk muscle mass ${ }^{(4)}$. Assessment of multiple sites enabled a comprehensive understanding of the association.

Several limitations of this study should be mentioned. First, the associations derived from a cross-sectional study do not necessarily indicate causality, although adults generally maintain stable long-term dietary habits, and the mean dietary value of the data from two surveys administered about 3 years apart was used for most of the participants. Moreover, as with any observational studies, despite attempts to control major potential confounders, the effect of residual confounding or unmeasured factors could not be completely ruled out. Third, volunteers were recruited to the study, which might have meant that the study population led a healthier lifestyle than the general population. However, the favourable associations did not significantly change according to age, education, income or dietary intake of energy and protein, suggesting that the generalisability of the results should not be greatly affected by these factors. Fourth, thought the aMDS are relatively easier to use, and is comparable among different populations, but is not targeted to our study population. Further modifications might be needed to improve its specificity in the Chinese people. Finally, the 649 withdrew subjects had longer YSM and lower physical activity at baseline than those 2520 participants followed-up (online Supplementary Table S5). We therefore could not exclude the possibility of lost-to-follow-up bias in this study.

\section{Conclusions}

The findings of this study show that aMDS positively associated with SMI in Chinese adults, particular males and younger subjects aged less than the medians of $59 \cdot 2$ and $62 \cdot 2$ years in females and males, suggesting that increasing the aMDS might be of great utility in the maintenance of muscle mass at middle ages. Additional longitudinal studies, particularly interventional studies, are needed to confirm these findings.

\section{Acknowledgements}

The authors thank the voluntary blood donors that participated in this study and are grateful for the help of other staff, postgraduates and undergraduates involved in data collection in this study.

This study was jointly supported by the National Natural Science Foundation of China (nos 81273049 and 81472965) and by the 5010 Program for Clinical Researches (no. 2007032) of the Sun Yat-Sen University (Guangzhou, China). The funding sources had no role in the design, analysis or writing of this article.

Y.-m. C. conceived and designed the study, and critically revised the paper; H.-y. T. analysed the data and wrote the paper; H.-y. T., R. Q., L.-p. J., Z.-y. C. and G.-d. C. collected the data; Y.-m. C. had primary responsibility for the final content. All the authors read and approved the final version of the manuscript.

The authors declare that there are no conflicts of interest.

\section{Supplementary material}

For supplementary material/s referred to in this article, please visit https://doi.org/10.1017/S0007114517001118

\section{References}

1. Devries MC \& Phillips SM (2015) Supplemental protein in support of muscle mass and health: advantage whey. $J$ Food Sci 80, Suppl. 1, A8-A15.

2. Morley JE, Anker SD \& von Haehling S (2014) Prevalence, incidence, and clinical impact of sarcopenia: facts, numbers, and epidemiology-update 2014. I Cachexia Sarcopenia Muscle 5, 253-259.

3. Chuang SY, Chang HY, Lee MS, et al. (2014) Skeletal muscle mass and risk of death in an elderly population. Nutr Metab Cardiovasc Dis 24, 784-791.

4. Cruz-Jentoft AJ, Baeyens JP, Bauer JM, et al. (2010) Sarcopenia: European consensus on definition and diagnosis: report of the European Working Group on Sarcopenia in Older People. Age Ageing 39, 412-423.

5. Janssen I, Shepard DS, Katzmarzyk PT, et al. (2004) The healthcare costs of sarcopenia in the United States. $J \mathrm{Am}$ Geriatr Soc 52, 80-85.

6. Wu IC, Lin CC, Hsiung CA, et al. (2014) Epidemiology of sarcopenia among community-dwelling older adults in Taiwan: a pooled analysis for a broader adoption of sarcopenia assessments. Geriatr Gerontol Int 14, Suppl. 1, 52-60.

7. Sofi F, Cesari F, Abbate R, et al. (2008) Adherence to Mediterranean diet and health status: meta-analysis. BMJ 337, a1344.

8. Sofi F, Abbate R, Gensini GF, et al. (2010) Accruing evidence on benefits of adherence to the Mediterranean diet on health: an updated systematic review and meta-analysis. Am J Clin Nutr 92, 1189-1196.

9. Scarmeas N, Stern Y, Tang MX, et al. (2006) Mediterranean diet and risk for Alzheimer's disease. Ann Neurol 59, 912-921.

10. Scarmeas N, Stern Y, Mayeux R, et al. (2009) Mediterranean diet and mild cognitive impairment. Arch Neurol 66, 216-225.

11. Psaltopoulou T, Sergentanis TN, Panagiotakos DB, et al. (2013) Mediterranean diet, stroke, cognitive impairment, and depression: a meta-analysis. Ann Neurol 74, 580-591.

12. Couto E, Boffetta P, Lagiou $\mathrm{P}$, et al. (2011) Mediterranean dietary pattern and cancer risk in the EPIC cohort. Br J Cancer 104, 1493-1499.

13. Trichopoulou A, Bamia C, Lagiou P, et al. (2010) Conformity to traditional Mediterranean diet and breast cancer risk in the Greek EPIC (European Prospective Investigation into Cancer and Nutrition) cohort. Am J Clin Nutr 92, 620-625.

14. Kenfield SA, DuPre N, Richman EL, et al. (2014) Mediterranean diet and prostate cancer risk and mortality in the Health Professionals Follow-up Study. Eur Urol 65, 887-894.

15. Buckland G, Agudo A, Lujan L, et al. (2010) Adherence to a Mediterranean diet and risk of gastric adenocarcinoma within the European Prospective Investigation into Cancer and Nutrition (EPIC) cohort study. Am J Clin Nutr 91, 381-390.

16. Steffl M, Bohannon RW, Petr M, et al. (2016) Alcohol consumption as a risk factor for sarcopenia - a meta-analysis. BMC Geriatr 16, 99.

17. Liang C, Oest ME, Jones JC, et al. (2009) Gestational high saturated fat diet alters $\mathrm{C} 57 \mathrm{BL} / 6$ mouse perinatal skeletal formation. Birth Defects Res B Dev Reprod Toxicol 86, 362-369. 
18. Smith GI, Julliand S, Reeds DN, et al. (2015) Fish oil-derived $n$-3 PUFA therapy increases muscle mass and function in healthy older adults. Am J Clin Nutr 102, 115-122.

19. Kim J, Lee Y, Kye S, et al. (2015) Association of vegetables and fruits consumption with sarcopenia in older adults: the Fourth Korea National Health and Nutrition Examination Survey. Age Ageing 44, 96-102.

20. Chrysohoou C, Panagiotakos DB, Pitsavos C, et al. (2004) Adherence to the Mediterranean diet attenuates inflammation and coagulation process in healthy adults: The ATTICA Study. J Am Coll Cardiol 44, 152-158.

21. Pastori D, Carnevale R, Bartimoccia S, et al. (2015) Does Mediterranean diet reduce cardiovascular events and oxidative stress in atrial fibrillation? Antioxid Redox Signal 23, 682-687.

22. Kelaiditi E, Jennings A, Steves CJ, et al. (2016) Measurements of skeletal muscle mass and power are positively related to a Mediterranean dietary pattern in women. Osteoporos Int 27, 3251-3260.

23. Nikolov J, Spira D, Aleksandrova K, et al. (2015) Adherence to a Mediterranean-style diet and appendicular lean mass in community-dwelling older people: results from the Berlin Aging Study II. J Gerontol A Biol Sci Med Sci 71, 1315-1321.

24. Hashemi R, Motlagh AD, Heshmat R, et al. (2015) Diet and its relationship to sarcopenia in community dwelling Iranian elderly: a cross sectional study. Nutrition 31, 97-104.

25. Chan R, Leung J \& Woo J (2016) A prospective cohort study to examine the association between dietary patterns and sarcopenia in Chinese community-dwelling older people in Hong Kong. J Am Med Dir Assoc 17, 336-342.

26. Zhang ZQ, He LP, Liu YH, et al. (2014) Association between dietary intake of flavonoid and bone mineral density in middle aged and elderly Chinese women and men. Osteoporos Int $\mathbf{2 5}$, $2417-2425$.

27. Zhang ZQ, Deng J, He LP, et al. (2013) Comparison of various anthropometric and body fat indices in identifying cardiometabolic disturbances in Chinese men and women. PLOS ONE 8, e70893.

28. Zeng FF, Xue WQ, Cao WT, et al. (2014) Diet-quality scores and risk of hip fractures in elderly urban Chinese in Guangdong, China: a case-control study. Osteoporos Int 25, 2131-2141.

29. Hu FB, Stampfer MJ, Rimm E, et al. (1999) Dietary fat and coronary heart disease: a comparison of approaches for adjusting for total energy intake and modeling repeated dietary measurements. Am J Epidemiol 149, 531-540.

30. Larsson SC, Bergkvist L \& Wolk A (2009) Long-term dietary calcium intake and breast cancer risk in a prospective cohort of women. Am J Clin Nutr 89, 277-282.
31. Trichopoulou A, Kouris-Blazos A, Wahlqvist ML, et al. (1995) Diet and overall survival in elderly people. BMJ 311, $1457-1460$.

32. Fung TT, Rexrode KM, Mantzoros CS, et al. (2009) Mediterranean diet and incidence of and mortality from coronary heart disease and stroke in women. Circulation 119, 1093-1100.

33. Murphy RA, Mourtzakis M, Chu QS, et al. (2011) Nutritional intervention with fish oil provides a benefit over standard of care for weight and skeletal muscle mass in patients with nonsmall cell lung cancer receiving chemotherapy. Cancer 117, 1775-1782.

34. Jacques H, Leblanc N, Papineau R, et al. (2010) Peanut protein reduces body protein mass and alters skeletal muscle contractile properties and lipid metabolism in rats. Br J Nutr $\mathbf{1 0 3}$, $1331-1339$.

35. Hintze J (2011) PASS 11. Kaysville, UT: NCSS, LLC. www.ncss.com

36. Slattery ML (2008) Defining dietary consumption: is the sum greater than its parts? Am J Clin Nutr 88, 14-15.

37. Lightfoot AP, McCormick R, Nye GA, et al. (2014) Mechanisms of skeletal muscle ageing; avenues for therapeutic intervention. Curr Opin Pharmacol 16, 116-121.

38. Cortright RN \& Koves TR (2000) Sex differences in substrate metabolism and energy homeostasis. Can J Appl Physiol 25, 288-311.

39. Hughes VA, Frontera WR, Roubenoff R, et al. (2002) Longitudinal changes in body composition in older men and women: role of body weight change and physical activity. Am J Clin Nutr 76, 473-481.

40. Gallagher D, Ruts E, Visser M, et al. (2000) Weight stability masks sarcopenia in elderly men and women. Am J Physiol Endocrinol Metab 279, E366-E375.

41. Fantin F, Di Francesco V, Fontana G, et al. (2007) Longitudinal body composition changes in old men and women: interrelationships with worsening disability. J Gerontol A Biol Sci Med Sci 62, 1375-1381.

42. Fano G, Mecocci P, Vecchiet J, et al. (2001) Age and sex influence on oxidative damage and functional status in human skeletal muscle. J Muscle Res Cell Motil 22, 345-351.

43. Kim J, Wang Z, Heymsfield SB, et al. (2002) Total-body skeletal muscle mass: estimation by a new dual-energy X-ray absorptiometry method. Am J Clin Nutr 76, 378-383.

44. Visser M, Fuerst T, Lang T, et al. (1999) Validity of fan-beam dual-energy X-ray absorptiometry for measuring fat-free mass and leg muscle mass. Health, Aging, and Body Composition Study - Dual-Energy X-ray Absorptiometry and Body Composition Working Group. J Appl Physiol (1985) 87, 1513-1520. 